\title{
Tunnel junction I(V) characteristics: review and a new model for $p-n$ homojunctions
}

N. Moulin, ${ }^{1}$ M. Amara, ${ }^{1, a)}$ F. Mandorlo, ${ }^{1, b)}$ and M. Lemiti ${ }^{1, c)}$

University of Lyon, Lyon Institute of Nanotechnology (INL) UMR CNRS 5270, INSA de Lyon, Villeurbanne, F-69621, FRANCE

(Dated: 1 December 2020)

Despite the widespread use of tunnel junctions in high-efficiency devices (e.g., multijunction solar cells, tunnel field effect transistors, and resonant tunneling diodes), simulating their behavior still remains a challenge. This paper presents a new model to complete that of Karlovsky and simulate an $I(V)$ characteristic of an Esaki tunnel junction. A review of different analytical models of band-to-band tunneling models is first presented. As a complement to previous work on tunnel junction simulation, the transmission coefficient is precisely determined and incorporated, the valley current between the tunneling and drift regimes is included, and calculations of physical parameters are updated. It is found that the model works for a broad range of values of the forward bias.

Keywords: tunnel junction, simulation, model

\section{NOMENCLATURE}

\section{Acronyms}

BTBT Band-to-band tunneling

TAT Trap-assisted tunneling

TC Transmission Coefficient

$T M \quad$ Transfer Matrix method

$W K B$ Wentzel Kramers Brillouin method

\section{Physical Constants}

$\hbar \quad$ Planck constant $/ 2 \pi$

c $\quad$ Speed of light in vacuum

$h \quad$ Planck constant

$k_{B} \quad$ Boltzmann constant

$q \quad$ Charge of the electron

\section{Variables}

$\theta \quad$ Parameter in Chynoweth's model

$D_{v} \quad$ Volume density of occupied levels above $E_{V}$

$E \quad$ Energy of an electron

$E_{C} \quad$ Energy of conduction band

\footnotetext{
a) Electronic mail: mohamed.amara@insa-lyon.fr

b) Electronic mail: fabien.mandorlo@insa-lyon.fr

c)Electronic mail: mustapha.lemiti@insa-lyon.fr
}

$E_{g} \quad$ Band gap

$E_{t} \quad$ Carrier transverse energy

$E_{V} \quad$ Energy of valence band

$E_{F n}, E_{F p}$ Fermi levels on $\mathrm{n}$ and $\mathrm{p}$ sides

$F \quad$ Electric field

$I_{P} \quad$ Peak tunnel current

$I_{V} \quad$ Valley current

$I_{B T B T}$ Tunneling current

$I_{C h} \quad$ Excess tunnel current

$k_{1} \quad$ Wave vector of an electron of energy $E$

$m_{C}, m_{V}$ Effective mass of the electron on the conduction, valence band

$m_{e} \quad$ Effective mass of the electron

$N_{D}, N_{A}$ Density of donor, acceptor impurity atoms

$n_{i} \quad$ Intrinsic carriers density

$S \quad$ Junction surface

T Temperature

$V \quad$ Applied bias

$V_{0} \quad$ Barrier height

$V_{P} \quad$ Voltage at $I=I_{P}$

$V_{V} \quad$ Voltage at $I=I_{V}$

$V_{b i} \quad$ Built-in voltage

$W \quad$ Free charge space

$W_{N}, W_{P}$ Depletion zone on $\mathrm{n}, \mathrm{p}$ side 


\section{INTRODUCTION}

The tunneling effect is a quantum phenomenon that allows carriers to cross a potential barrier without jumping over it. If the barrier is thin enough and empty sites are available in the right range of energies, carriers from one side can tunnel to these empty sites. It should be noted that although the word "tunnel" carries the image of going through, it is rather the case that carriers disappear and then reappear on the other side of the barrier. The probability for this phenomenon to occur is highly correlated with the width of the barrier and the energy of the carrier. There are many devices $\left(\right.$ TFETs $^{1}$, $\operatorname{RTDs}^{2} \ldots$ ) that rely on this effect for their properties, with specific materials and doping levels being chosen to create a tunnel effect. For example, in the field of photovoltaics, tandem solar cells score the highest efficiency on both laboratory and industrial scales by using tunnel junctions as key parts of their structures linking different subcells. $^{3}$

However, there have been few studies focusing on the behavior of this particular component of these cells, and indeed detailed simulations conducted by Hermle et al. ${ }^{4}$ and Liu et al. ${ }^{5}$ have highlighted the difficulty of such a task. A reliable tunneling model should be able to simulate three different regimes: the peak current, where tunneling is dominant; the valley current, where the tunneling probability is low and drifting starts to occur; ${ }^{6,7}$ and the diode regime, where drifting is dominant. Yajima and Esaki discovered the tunneling effect experimentally in a highly doped germanium diode ${ }^{8}$ in 1958, and Esaki ${ }^{9}$ proposed a model to describe it. In 1960, an extensive theory was developed by Kane, ${ }^{6}$ following a suggestion from Zener about a tunneling phenomenon. Soon after, Karlovsky ${ }^{10}$ proposed a simpler version of the Esaki model that was valid as long as the difference between the gap and the Fermi levels was small enough. In 1969, the first review of tunneling models was presented by Duke. ${ }^{11}$ In his book, he compared several models and approaches to establish which of these was the most accurate at that time. He considered, among others, the previous work by Kane and Esaki, as well as that by Keldysh, ${ }^{12}$ who had found the same expression as Kane, although they had worked independently because of the lack of scientific communication between East and West during the Cold War.

As research on semiconductors progressed, several models were proposed ( $\mathrm{p}-\mathrm{n}$ diode, CMOS, SOI, III-V, etc.). In 1989, at a conference in Berlin, Hurkx ${ }^{13}$ presented a new model of the recombination rate based on the 1952 work of Shockley and Read ${ }^{14}$ on Shockley-ReadHall (SRH) recombination. This model was based on trap-assisted tunneling (TAT) associated with SRH recombination and band-to-band tunneling (BTBT) at reverse bias. In his paper, Hurkx calculated the contribution of the tunneling effect as a recombination rate instead of a current density. Along the same lines, in 1991, Klaassen ${ }^{15}$ presented a model that was also mainly de- veloped for TAT, since he considered this to be dominant over direct tunneling at forward-bias polarization.

Hurkx's work was tested in 2008 by Baudrit and Algora, ${ }^{16}$ who underlined the fact that Hurkx's model does not work at forward bias. Currently, the reference model remains that of Tsu and Esaki. ${ }^{17}$. Hermle et al. ${ }^{4}$ presented a method to simulate an isolated GaAs tunnel junction at forward bias.

The first part of the present paper compares these analytical models in the context of simulation of direct BTBT. As far as the simulation method is concerned, a review of different analytical models and simulations leads to the conclusion that the nonlocal approach (see Fig. 1 of Ref. 5) is the most precise, ${ }^{4,5,18}$ since it considers effective carrier transport. Also, only BTBT need be considered as the tunneling regime, since it is sufficient for correct simulation of an $I(V)$ curve. $^{4,5}$ The tunneling probability can be calculated using the transfer matrix method, since this method has proven to be accurate ${ }^{5}$ and is computationally less expensive than the Wentzel-KramersBrillouin (WKB) method. ${ }^{19}$ Simulations are run under Matlab software.

The main goal of this work is to construct an analytical model that is able to calculate $I(V)$ curves to aid in the design of the doping levels and dimensions of a tunnel diode. As the experimental work of the present authors is mainly concerned with silicon-based diodes, the review will focus on models that can be applied to this material.

\section{REVIEW OF TUNNEL JUNCTION MODELS}

\section{A. Tsu-Esaki based models}

\section{Tsu-Esaki model}

This model ${ }^{17}$ is based on a previous one-dimensional (1D) superlattice model published in $1971^{20}$. The 1973 model incorporates a finite number of periods and a short electron mean free path. It also applies to multibarrier tunneling. The effective mass is calculated for unperturbed structures, and the 3D Schrödinger equation is solved for a $1 \mathrm{D}$ periodic potential $V$. Making the simplifying assumption that the transmission coefficient $T C$ is a function of $k_{l}$ only (the wave vector lies along the barrier dimension), we obtain

$$
\begin{aligned}
& J= \\
& \frac{q m_{e} k_{B} T}{2 \pi^{2} \hbar^{3}} \int_{0}^{\infty} T C\left(E_{l}\right) \cdot \ln \left(\frac{1+\exp \left(\frac{E_{F n}-E_{l}}{k_{B} T}\right)}{1+\exp \left(\frac{E_{F p}-E_{l}-q V}{k_{B} T}\right)}\right) d E_{l},
\end{aligned}
$$

where $m_{e}$ is the electron effective mass and $E_{l}$ is the electron energy along $k_{l}$. This expression is general and 
holds regardless of the type of semiconductor (direct or indirect bandgap).

\section{Kane model}

In 1961, Kane developed a model ${ }^{21}$ similar to Esaki and the later Karlovsky models, but using a different approach. This theory was adapted from Keldysh's ${ }^{12}$ theory of indirect tunneling supplied by phonons. No transmission coefficient is calculated, but instead the model is based on a function of the effective density of states, $D_{\text {Kane }}$, given by

$$
\begin{aligned}
D_{\text {Kane }}= & \int\left[f_{1}\left(E_{1}\right)-f_{2}\left(E_{2}\right)\right]\left[1-\exp \left(-\frac{E_{1}}{E_{t}}\right)\right] \\
& \times\left[1-\exp \left(-\frac{E_{2}}{E_{t}}\right)\right] d E
\end{aligned}
$$

This function is zero when the electron has an energy outside the Fermi levels. Several subsequent models ${ }^{15,22}$ have adopted similar functions. In Eq. (2), $E_{t}$ is the transverse component of the electron energy, and subscripts 1 and 2 refer to the bands before and after tunneling. When a bias is applied, three regimes can be distinguished by comparing $q V$ with the carrier energy. The tunneling current characteristic is thus mainly monitored by the function $D_{\text {Kane }}$, which takes into account the effect of phonons on the indirect tunneling process.

This model deals with the cases of direct and indirect semiconductors differently. As Si is of the latter type, we will focus on the corresponding model for our review.

\section{Karlovsky model}

Karlovsky ${ }^{10}$ proposed a simple expression for the tunnel current in an Esaki diode based on the Esaki model:

$$
\begin{aligned}
& E_{1}=E_{F n}-E_{C}, E_{2}=E_{V}-E_{F p}, \\
& J=\frac{A}{S} \int_{E_{C}}^{E_{V}} \frac{E_{F n}-E_{F p}}{4 k_{B} T} \sqrt{\left(E-E_{C}\right)\left(E_{V}-E\right)} d E,
\end{aligned}
$$

where $S$ is the surface area of the junction. This expression is valid if the distances between the Fermi levels and the edge bands $\left(E_{1}\right.$ and $\left.E_{2}\right)$ are small $\left(\leq 2 k_{B} T\right)$. With $E_{F n}-E_{F p}=q V$, this expression becomes

$$
J=\frac{A}{S} \cdot q V\left(E_{1}+E_{2}-q V\right)^{2},
$$

which is a polynomial function depending on the bias and the Fermi levels only. However, an uncertainty remains about the expression for $A$, which is not given explicitly in the original paper. By comparison with other models, it can be considered as a scaling factor.

\section{Duke model}

In 1969, Duke $^{11}$ presented what was then the state of art in tunneling theory, describing all the main tunneling models, their weak points, and their advantages.

The model simulated in this review is the one described as the most accurate at that period. In this model, the barrier profile is corrected by a coefficient introduced into the expression for the maximum electric field. However, it does not provide an accurate expression for the current when there is a high impurity concentration and it tends to overestimate the valley current under a forward bias.

\section{B. Recombination based models}

\section{Hurkx model}

Hurkx $^{22}$ chose to calculate a recombination contribution rather than a tunnel current, using the following expression for BTBT:

$R_{B T B T}=-B|F|^{\sigma} D_{\text {Kane }} \exp \left(\frac{-F}{F_{0}}\right)$,

where $F$ is the local electric field. $\sigma$ is a coefficient that is equal to 2 for direct tunneling and 2.5 for indirect tunneling (as in the case of silicon ). DKane is calculated according to the theory of Keldysh ${ }^{12}$ and Kane, ${ }^{21}$ except that in the case of null and reverse bias, Hurkx proposes the following simpler expression:

$$
D_{H u r k x}=\frac{1}{\exp \left(\frac{\left.-E_{F p}-q V\right)}{k_{B} T}\right)+1}-\frac{1}{\exp \left(\frac{-E_{F n}-q V}{k_{B} T}\right)+1} .
$$

This function is equal to 1 when the electron energy is between $E_{F n}$ and $E_{F p}$ and 0 elsewhere.

This approach corresponds to what is known as the local tunneling model. Its drawbacks compared with the nonlocal approach in the case of tunneling theory were also pointed out by Hermle et al. ${ }^{4}$

\section{Klaassen model}

The Klaassen model is focused mainly on TAT. In the same way as in the Hurkx model, the tunneling contribution is calculated as a recombination factor $R$ instead of a current density. The total tunneling effect is composed of two contributions: direct tunneling with $R_{B T B T}$ given by Eq. (5)) and a TAT mechanism at forward and reverse bias with

$$
R=\frac{n_{t} p_{t}-r_{n} r_{p} n_{l}^{2}}{\tau_{p}\left(n_{t}+r_{n} n_{l}\right)+\tau_{n}\left(p_{t}+r_{p} n_{l}\right)}-R_{B T B T},
$$

where $r_{n}$ and $r_{p}$ are emission probabilities, $n_{t}$ and $p_{t}$ are the concentrations of tunneling carriers in the depletion 
region, and $\tau_{n}$ and $\tau_{p}$ are capture rates. The direct tunneling current density is then obtained by integrating the recombination factor:

$$
R_{B T B T}=-\frac{d J}{d E} \cdot F
$$

\section{COMPARISON OF TUNNEL JUNCTION MODELS}

\section{A. Method and common parameters}

The methods of calculation for material parameters (e.g., effective mass and intrinsic carrier concentration) and the ways in which physical phenomena (e.g., bandgap narrowing and the transmission coefficient) are taken into account differ among the models. Therefore, to allow a proper comparison, it is necessary step to fix the calculation methods for both known and new physical parameters.

This work compares the models for a silicon $\mathrm{p}-\mathrm{n}$ junction (without any oxide layer) at a high doping level.

One of the key parameters in a tunneling model is the transmission coefficient, which gives the probability for an electron at energy $E$ to tunnel through a potential barrier of height $V_{0}$ and thickness $a$. The higher and thicker the barrier, the less probable it is that tunneling occurs. Accordingly, the higher the energy $E$, the more probable will tunneling be. Therefore, for a given height, there is a limiting thickness from which the probability starts to become negligible. Similarly, it is possible to determine a limiting energy from which electron tunneling is possible.

To calculate the tunneling probability, several methods have been developed, of which the two main ones are the Wentzel-Kramers-Brillouin (WKB) method and the transfer matrix method (TM). We have chosen to use the latter here, since it is more stable with respect to the geometry of the barrier and is computationally less expensive. ${ }^{19}$ We use values of common physical parameters at $300 \mathrm{~K}$ as calculated by the most up-to-date methods (Table I).

\section{B. Model comparison: analysis}

With the parameters listed in Table I, the current density curves are calculated for each model in forward bias (Fig. 1). What we expect, is the typical " $\mathrm{S}$ " shape of a tunneling $\mathrm{I}(\mathrm{V})$ curve.

- The Hurkx model works only for reverse bias ${ }^{16}$ and therefore does not appear in the simulation for forward bias.

- The Tsu-Esaki and Duke models give a very low current density, and the results do not exhibit a tunneling peak at forward bias with these parameters, contrary to 1970 curves. ${ }^{17}$

\begin{tabular}{llll} 
Name & Value & Unit & Reference \\
\hline$T$ & 300 & $\mathrm{~K}$ & \\
$N_{C}(300)$ & $2.89 \times 10^{19} \mathrm{~cm}^{-3}$ & Couderc et $a .^{23}$ \\
$N_{V}(300)$ & $1.04 \times 10^{19} \mathrm{~cm}^{-3}$ & Couderc et al. $^{23}$ \\
$n_{i 0}(300)$ & $9.65 \times 10^{19} \mathrm{~cm}^{-3}$ & Couderc et $a .^{23}$ \\
$E_{A}$ & $45 \times 10^{-3}$ & $\mathrm{eV}$ & Sze et $\mathrm{Ng}^{24}$ \\
$E_{D}$ & $44 \times 10^{-3}$ & $\mathrm{eV}$ & Sze et $\mathrm{Ng}^{24}$ \\
BGN effect & $\Delta E_{G}$ & $\mathrm{eV}$ & Schenk et al. ${ }^{25}$ \\
Effective masses & $m_{c}, m_{v}$ & $\mathrm{~kg}$ & Couderc et al. ${ }^{23}$ \\
$N_{A}$ & $8 \times 10^{19}$ & $\mathrm{~cm}{ }^{-3}$ & \\
$N_{D}$ & $2 \times 10^{20}$ & $\mathrm{~cm}^{-3}$ & \\
$S$ & 64 & $\mu \mathrm{m}^{2}$ & \\
$V$ & {$[-0.9,0.5]$} & $\mathrm{V}$ & \\
\hline
\end{tabular}

TABLE I: Default parameters and references for their calculation.

- The Klaassen and Karlovsky models give an average current density, and the results exhibit a tunneling peak at forward bias. However, the Klaassen model does not apply after the peak, since the coefficient $D_{\text {Kane }}$ is zero outside the tunneling regime [Eq. (2)].

In conclusion, this comparison reveals that the Karlovsky model gives the best shape for the tunneling $I(V)$ curve. However, it is incomplete and does not take into account important physical phenomena such as that represented by the transmission coefficient. Also, it does not cover the valley regime.

\section{NEW TUNNELING MODEL}

\section{A. General description}

A new model to complete that of Karlovsky will be developed here by considering additional contributions from the tunneling current and the excess current. Each of these currents is linked to a specific type of carrier transfer. For the first, we consider a pure BTBT regime expressed by a third-degree polynomial $P_{3}$ weighted with a detailed $T C$. For the second, we consider a transition from a tunneling regime to a drift regime, using a TAT model developed by Chynoweth et al.: ${ }^{7} I_{C h}$. This approach is similar to a semiempirical one developed by Demassa and Knott ${ }^{26}$ and completed by $\mathrm{Roy}^{27}$ in the early 1970s. Two scaling factors $A$ and $A^{\prime}$ are introduced to balance the two contributions:

$$
I=A \cdot T C \cdot P_{3}+A^{\prime} \cdot I_{C h}
$$




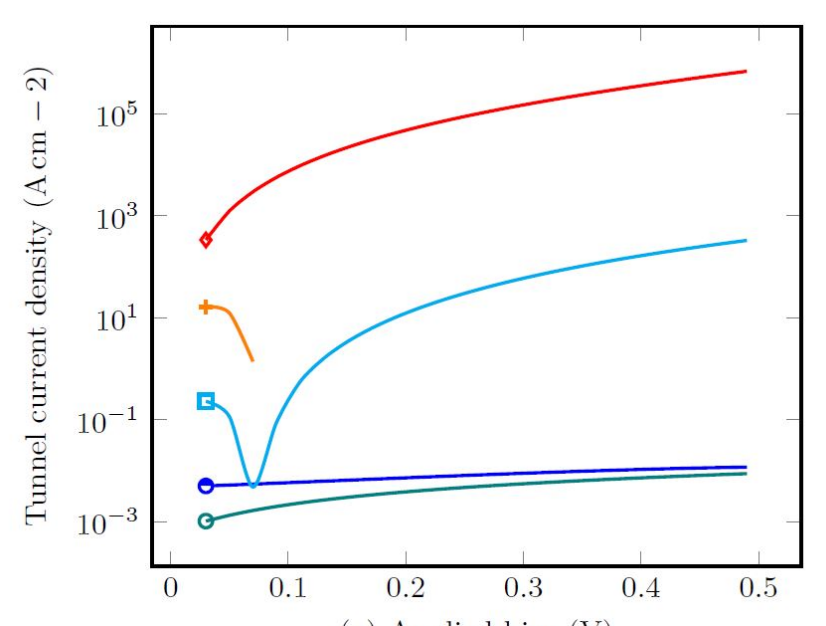

(a) Applied bias (V)
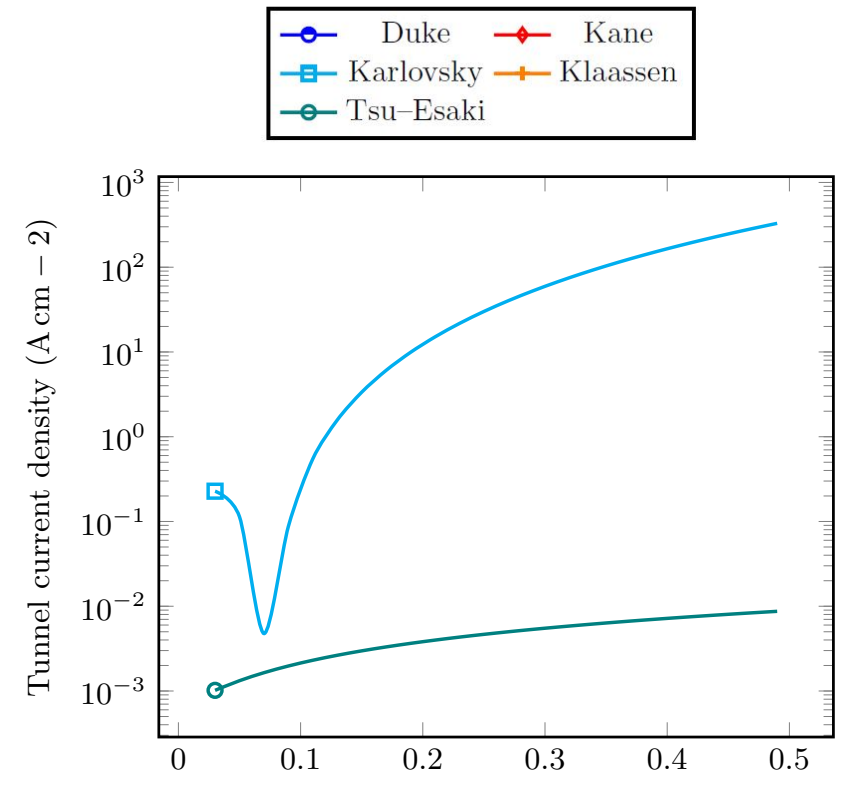

(a) Applied bias (V)

—-Karlovsky - - Tsu-Esaki

FIG. 1: Comparison of direct tunneling models at forward bias.

\section{B. Transmission Coefficient}

The simulation of a tunnel current is strongly linked with the transmission coefficient $T C$. This parameter gives the probability for an electron at a given energy to cross the junction. Among the various methods commonly used to calculate $T C$, we have chosen the TM method, since, as already mentioned, it is easy to implement and computationally less expensive. ${ }^{19}$

The standard TM method relies on two expressions: ${ }^{28}$

$$
E<q V_{0}: T C=\frac{1}{1+\frac{\left(q V_{0}\right)^{2} \sinh ^{2}\left(k_{1} W\right)}{4 E\left(q V_{0}-E\right)}}
$$

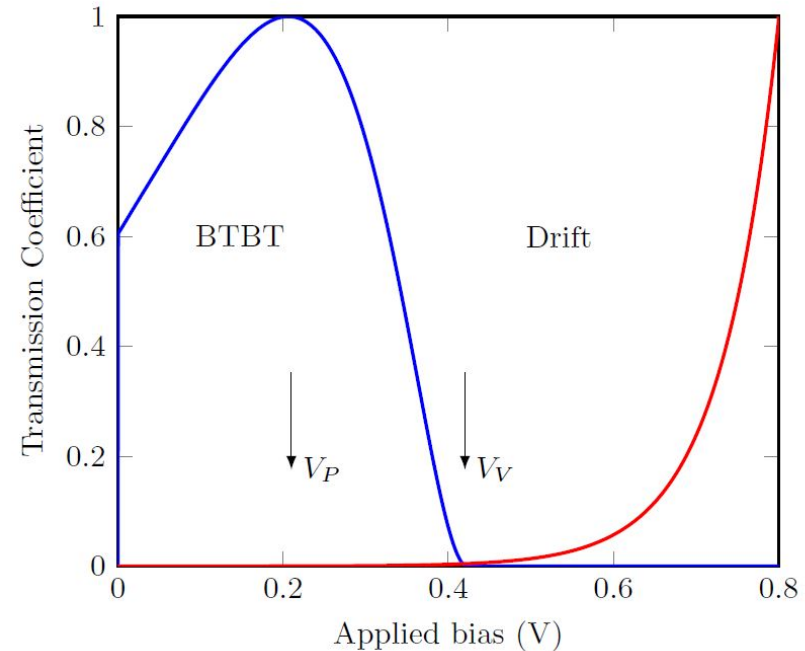

FIG. 2: Calculation of $T C$ for tunneling (blue) and drift (red) regimes. The two curves have been normalized separately.

$$
\begin{gathered}
E>q V_{0}: T C=\frac{1}{1+\frac{\left(q V_{0}\right)^{2} \sin ^{2}\left(k_{1} W\right)}{4 E\left(E-q V_{0}\right)}} \\
k_{1}=\sqrt{\frac{2 m_{e}\left|q V_{0}-E\right|}{\hbar^{2}}}
\end{gathered}
$$

Equation (10)) calculates $T C$ for electrons that can tunnel through a barrier of height $V_{0}$, whereas Eq. (11)) calculates $T C$ for electrons that can drift over the barrier (Fig. 2). The key task is to sort out those electrons that tunnel from those that drift over the barrier.

To understand how we incorporate $T C$ into our model, it is necessary to understand what happens to the current between zero and the peak tunneling current $I_{P}\left(V_{P}\right)$, between $I_{P}$ and the valley current $I_{V}\left(V_{V}\right)$, and after $I_{V}$ (Fig. 3a). When $0<V<V_{P}$, the Fermi levels separate, and the occupied states come before the free states. With high doping levels, the barrier between the two sides of the junction is thin enough for tunneling to occur (Fig. 3b). It should be noted that the BTBT regime is theoretically limited by $E_{F p}$ at the bottom and $E_{F n}$ at the top (in practice, a few carriers can be found above $E_{F n}$, and BTBT is limited by $E_{V}$ on the p side). In Fig. 2, the blue curve that shows the tunneling $T C$ increases with the bias until it reaches a maximum (which corresponds to $V=V_{P}$ ). The red curve shows that almost no drift occurs at these values of bias.

When $V_{P}<V<V_{V}$, fewer and fewer states are located before the free states and can tunnel. Meanwhile, higher-energy states can no longer tunnel, and drifting starts to occur (Fig. 3c). In Fig. 2, the tunneling $T C$ decreases after $V_{P}$, and the drift $T C$ starts to increase, revealing the transition from one regime to the other. At $V_{V}<V$, some occupied states are located above $E_{V}$ and can no longer tunnel except by using traps located in the 


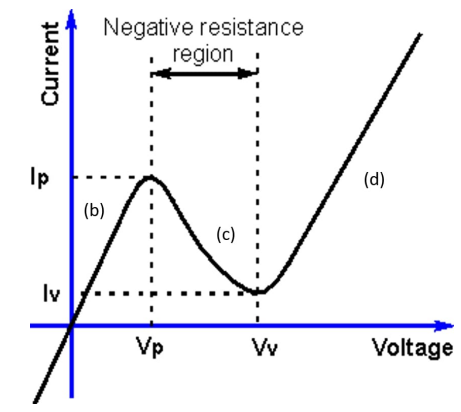

(a)

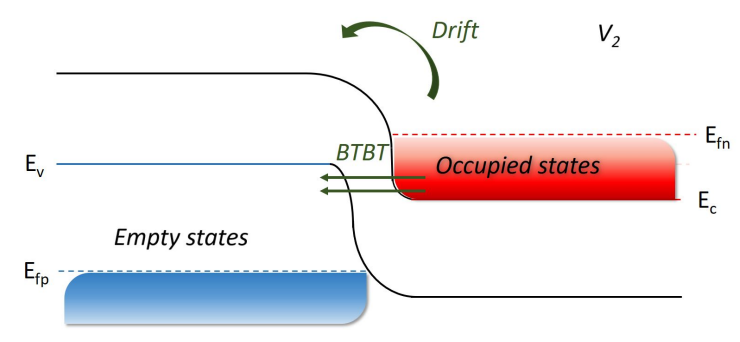

(c)

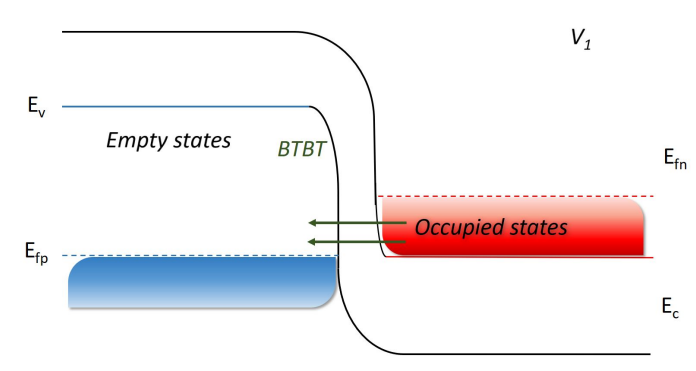

(b)

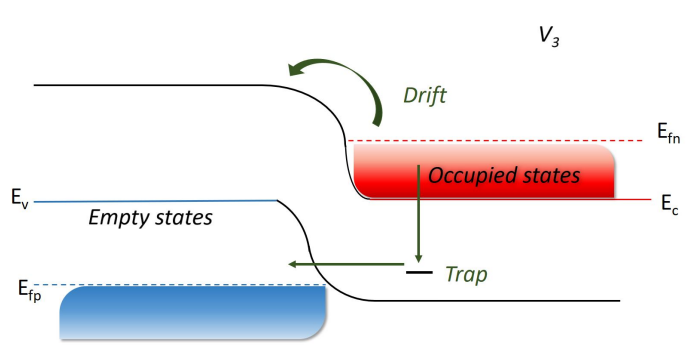

(d)

FIG. 3: Schematic representation of the evolution of a tunnel junction when a forward bias is applied. (a) Ideal tunnel diode $I(V)$ curve. $^{29}$ (b) Band diagram at low forward bias below $V_{P}$. (c) Band diagram at higher forward bias between $V_{P}$ and $V_{V}$. (d) Band diagram at high forward bias above $V_{V}$.

forbidden gap. However, more carriers with enough energy can drift over the barrier (Fig. 3d). In Fig. 2, the blue curve is almost at zero, while the red curve begins to grow significantly.

Therefore, during the simulation, for each bias, we sort out two categories of electrons and apply the appropriate relation from Eq. (10) or Eq. (11). Following this, only the BTBT contribution to $T C$ is retained and incorporated into the calculation of the current. As the drift probability is much higher than the tunneling probability, separating the two contributions allows better precision for the tunneling part of the $I(V)$ curve (Fig. 2, red and blue curves).

\section{Tunneling regime}

At low forward bias, two key values are considered: the peak current $I_{P}$, where the tunneling regime is at its highest, and the valley current $I_{V}$, where tunneling is at its lowest. In this model, the tunneling current is composed of two terms: $T C$ and a third-degree polynomial $P_{3}$ inspired by the Karlovsky model:

$$
\begin{aligned}
& I_{B T B T}=T C \cdot P_{3}, \\
& \frac{\partial I_{B T B T}}{\partial V}=T C^{\prime}(V) \cdot P_{3}(V)+T C(V) \cdot P_{3}^{\prime}(V) .
\end{aligned}
$$

For there to be maxima at $V_{P}$ and $V_{V}, I_{B T B T}$ must satisfy the following conditions:

$$
\begin{aligned}
& \text { At } V=0: P_{3}(0)=0, \\
& \text { At } V=V_{P}: T C^{\prime}=0 \Rightarrow P_{3}^{\prime}\left(V_{P}\right)=0, \\
& \text { At } V=V_{V}: T C \approx 0 \Rightarrow P_{3}\left(V_{V}\right)=0 .
\end{aligned}
$$

In fact, the last condition is an approximation, since tunneling does not stop exactly at $V_{V}$ but at a slightly higher bias. However, for this first approximation, it allows a local minimum at the correct position. These three conditions give us the expressions for the polynomial coefficients:

$$
P_{3}=V \cdot\left(V^{2}-\nu_{p v} V+\nu_{p v} V_{V}-V_{V}^{2}\right)
$$

with

$$
\nu_{p v}=3 / 2\left(V_{V}+V_{P}\right)
$$

Modulo a scaling factor and $T C$, Eq. (17) gives the tunneling contribution to the $I(V)$ curve. We can see from the orange curve with the filled dot in Fig. 4 that the peak and valley tensions are aligned with the experimental data, but the slopes around $I_{P}$ do not match exactly.

\section{Valley current}

Because $T C$ is calculated only for the BTBT regime, the last part of the polynomial curve is zero (Fig. 4, blue 
curve with a square). To simulate the drift regime, we choose to add another contribution based on an expression from Chynoweth et al. ${ }^{7}$ that focuses mainly on the valley tunnel current:

$I_{C h}=D_{v} P$,

with

$P=\exp \left(-\frac{\alpha E_{t}^{3 / 2}}{F}\right)$,

$E_{t}=E_{G}-q V+E_{F n}+E_{F p}$

$F=2 \frac{\sqrt{V_{b i}-V}}{W}$

$\alpha=\theta \cdot 4 \frac{\sqrt{2 m_{e}}}{3 q \hbar}$, with $\theta \approx 1$

Here, $E_{t}$ is the energy barrier faced by the carrier (which is equal to the difference between the two sides of the junction), $F$ is the maximum field for a step junction, and $P$ is the probability for a carrier to cross the gap (which is similar to what has previously been called $T C$ ). $D_{v}$ is the volume density of occupied levels above the valence band for a given energy (i.e., the filled states in the defects for energies in the band gaps). For a pure material, this density will be very low, thus resulting in a low excess current.

The expression for the tunnel current including this valley current is

$$
I=A \cdot T C \cdot V\left(V^{2}-\nu_{p v} V+\nu_{p v} V_{V}-V_{V}^{2}\right)+A^{\prime} \cdot I_{C h}(\theta)
$$

The shape of the valley current depends mainly on $\theta$ (Fig. 5). Thus, the model has three input parameters $\left(N_{A}, N_{D}\right.$, and the surface area $S$ of the junction) and three fitting parameters (the scaling factors $A, A^{\prime}$, and $\theta$ ). With this expression, the valley current is nonzero (Fig. 4, black full curve) and the drift part of the curve is consistent with experimental values. The remaining discontinuity at the valley current is a consequence of the initial hypothesis which approximates $T C\left(V_{V}\right)$ to 0 (Eq.16).

The table below (Tab. II) lists the contributions of the new model compared to Karlovsky model.

\section{E. Effect of temperature}

As mentioned for the Karlovsky model, the hypotheses leading to the third-degree polynomial are valid as long as the widths of the Fermi levels are smaller than $2 k_{B} T$. An abscissa (Fig. 6) can be drawn to find the limiting doping level for each temperature. Below these limits, the hypotheses of the model are valid. Above them, the


FIG. 4: Progressive improvements in simulation using Yan's ${ }^{30}$ characteristic data for a silicon diode.

$N a=1 \times 10^{20} \mathrm{~cm}^{-3}, N d=1 \times 10^{20} \mathrm{~cm}^{-3}, T=300 \mathrm{~K}$

model can still be used, but with caution. For example, for junctions with high doping levels, the model is more accurate at high temperatures. The limits at $3 k_{B} T$ and $4 k_{B} T$ are quite far from $2 k_{B} T$ (Fig. 6 ), so exceeding the limit by $10 \%$ does not strongly affect the accuracy of the model.

In Fig. 7, the experimental data and the doping levels are the same as those considered in Sec. IV C, and the parameters are calculated for temperatures ranging from 100 to $500 \mathrm{~K}$. When the temperature increases, we can see that the positions of $V_{P}$ and $V_{V}$ do not vary. However, there is a small shift toward higher current at $V_{P}$ and a greater shift in current at $V_{V}$. These shifts have 
Karlovsky model New model

\begin{tabular}{lll}
\hline Tunneling peak & yes & yes \\
Valley fit & no & partial \\
Voltage range & {$\left[0, V_{V}\right]$} & {$\left[0, \gg V_{V}\right]$} \\
$T C$ & no & yes \\
Drift fit & no & yes \\
Band gap narrowing no & yes \\
\hline
\end{tabular}

TABLE II: Contributions of the new model compared to previous most accurate model



FIG. 5: Effect of $\theta$ on the shape of the valley current

been observed experimentally by Schmid et al., ${ }^{31}$ who found a significant vertical shift at $V_{V}$ for junctions with a peak-to-valley current ratio (PVCR) of 2.63. For junctions with a PVCR of 1.5 , there was a small shift at $V_{P}$ and a vertical shift at $V_{V}$. The junction considered in the present paper has a PVCR of 2.17. Therefore, the shifts observed in the simulation are consistent with the experimental curves described by Schmid et al. ${ }^{31}$

Experimental data is supposed to be measured at room temperature. This is confirmed by the fact that the best fit is obtained for the simulation at $300 \mathrm{~K}$ (cyan dashed line).

\section{CONCLUSIONS}

In this paper, different analytical models for a $\mathrm{p}-\mathrm{n}$ tunnel junction have been investigated and compared. This review has revealed that recombination models have a limited range of validity and are not easy to implement. A new model to complete that of Karlovsky has been proposed, with the following new aspects:

- accurate calculation of physical parameters taking

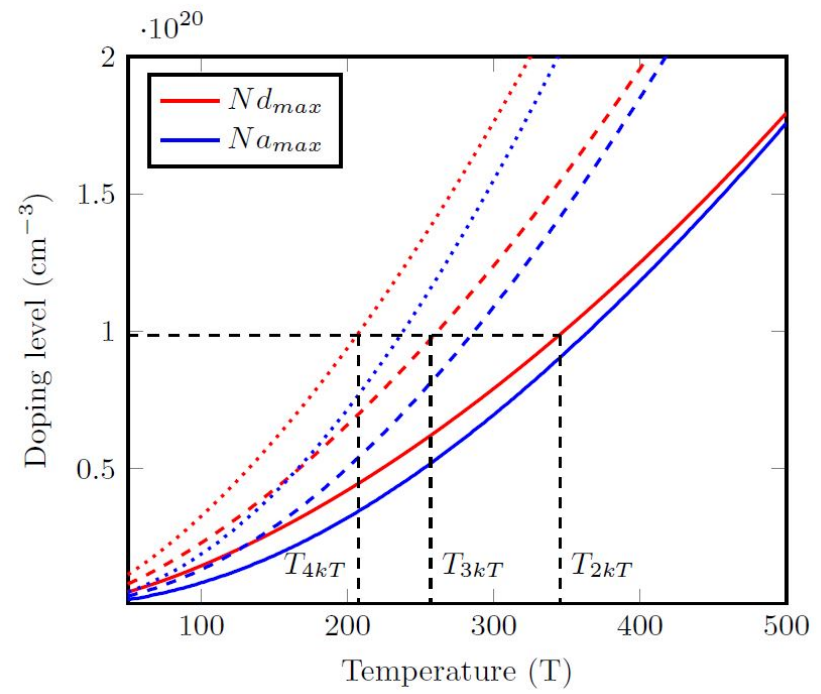

FIG. 6: Doping levels at which $E_{C}-E_{F n}$ and $E_{V}-E_{F p}$ are equal to $2 k_{B} T$ (solid curve), $3 k_{B} T$ (dashed curve), $4 k_{B} T$ (dotted curve) for silicon.
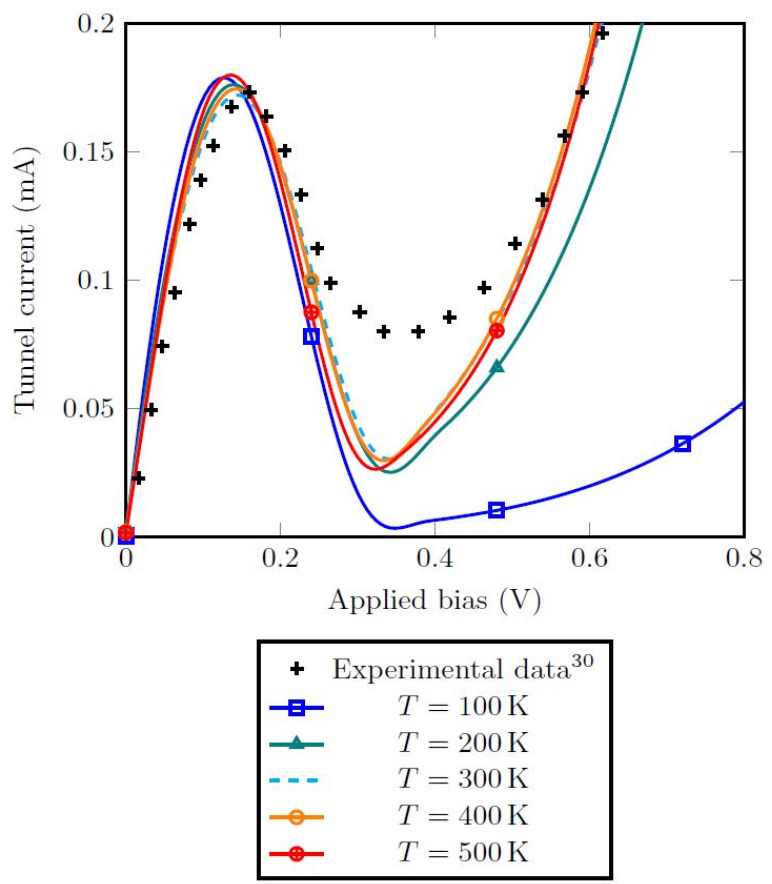

FIG. 7: Impact of temperature on a tunnel diode.

account of extra phenomena such as bandgap narrowing and degenerate Fermi levels;

- decomposition of the model into two contributions (tunneling and valley);

- incorporation of the transmission coefficient;

- incorporation of the valley regime using the model 


\section{of Chynoweth et al.;}

- prediction of temperature effects that are consistent with experimental results.

This model, valid at high temperature, shows a good correlation with experimental data.

In this work, only BTBT has been considered, since it is assumed to be dominant in materials with low impurity concentrations. Also, from the work of Hermle et al. ${ }^{4}$ it appears that the inclusion of a TAT mechanism does not lead to any significant changes in the simulated curves. Also, a nonlocal approach as recommended by Hermle et $a l .{ }^{4}$ has been adopted by modeling carrier transport through a transmission coefficient.

Further investigations could include finding a detailed expression for the valley current and extending this model to heterojunctions. The latter should considers other tunneling effects than pure BTBT and incorporate them in the calculation of $T C$. Energy band offset at the interface between the two materials may induce additionnal modifications.

\section{REFERENCES}

${ }^{1}$ Abadi,R., Saremi, M., "A resonant tunneling nanowire field effect transistor with physical contractions: a negative differential resistance device for low power very large scale integration applications," Journal of electronic materials 47, 1091-1098 (2017).

${ }^{2}$ Goharrizi, A., Zoghi, M., Saremi, M., "Armchair graphene nanoribbon resonant tunneling diodes using antidote and bn doping," IEEE Transactions on electron devices 63, 3761-3768 (2016).

${ }^{3}$ M. A. Green, "Intrinsic concentration, effective densities of states, and effective mass in silicon," Journal of Applied Physics 67, 2944-2954 (1990).

${ }^{4}$ M. Hermle, G. Ltay, S. P. Philipps, and A. W. Bett, "Numerical simulation of tunnel diodes for multijunction solar cells," Progress in Photovoltaics: Research and Applications 16, 409418 (2008-08).

${ }^{5}$ Y. Liu, M. Ahmadpour, J. Adam, J. Kjelstrup-Hansen, H.-G. Rubahn, and M. Madsen, "Modeling multijunction solar cells by nonlocal tunneling and subcell analysis," IEEE Journal of Photovoltaics 8, 1363-1369 (2018-09).

${ }^{6}$ E. O. Kane, "Zener tunneling in semiconductors," Journal of Physics and Chemistry of Solids 12, 181-188 (1960).

${ }^{7}$ A. G. Chynoweth, W. L. Feldmann, and R. A. Logan, "Excess tunnel current in silicon esaki junctions," Physical Review 121, 684 (1961).

${ }^{8}$ L. Esaki, "New phenomenon in narrow germanium pn junctions," Physical Review 109, 604-605 (1958).

${ }^{9}$ L. Esaki, "New phenomenon in narrow germanium p-n junctions," Physical Review 109, 604-605 (1958).

${ }^{10} \mathrm{~J}$. Karlovsky, "Simple method for calculating the tunneling current of an esaki diode," Physical review 127 (1962).

${ }^{11}$ C. Duke, Tunneling in Solids (Solid States of Physics, 1969).

${ }^{12}$ Keldysh, "Behaviour of non-metallic crystals in strong electric fields," Soviet Physics JETP 6 (1958).

${ }^{13}$ G. A. M. Hurkx, F. G. O'Hara, and M. P. G. Knuvers, "Modelling forward-biased tunneling," in Solid State Device Research Conference, 1989. (IEEE, 1989) pp. 793-796.

${ }^{14} \mathrm{~W}$. Shockley and W. T. Read Jr, "Statistics of the recombinations of holes and electrons," Physical review 87, 835 (1952).
${ }^{15}$ Simulation of semiconductor devices and process, Vol. Vol. 4 (W. Fichtner, D. Aemmer- Zurich (Switzerland), September 1214,1991).

${ }^{16} \mathrm{M}$. Baudrit and C. Algora, "Modeling of GaInP/GaAs dualjunction solar cells including tunnel junction," in Photovoltaic Specialists Conference, 2008. PVSC'08. 33rd IEEE (IEEE, 2008) pp. 1-5.

${ }^{17}$ R. Tsu and L. Esaki, "Tunneling in a finite superlattice," Applied Physics Letters 22, 562-564 (1973).

${ }^{18}$ M. Baudrit and C. Algora, "Tunnel Diode Modeling, Including Nonlocal Trap-Assisted Tunneling: A Focus on III-V Multijunction Solar Cell Simulation," IEEE Transactions on Electron Devices 57, 2564-2571 (2010).

${ }^{19}$ A. Gehring, "Simulation of tunneling in semiconductor devices." (1975).

${ }^{20} \mathrm{R}$. Tsu and L. Esaki, "Nonlinear optical response of conduction electrons in a superlattice," Applied Physics Letters 19, 246-248 (1971).

${ }^{21}$ E. O. Kane, "Theory of tunneling," Journal of Applied Physics 32, 83-91 (1961).

${ }^{22}$ G. A. M. Hurkx, D. B. M. Klaassen, and M. P. G. Knuvers, "A new recombination model for device simulation including tunneling," IEEE Transactions on electron devices 39, 331-338 (1992).

${ }^{23}$ R. Couderc, M. Amara, and M. Lemiti, "Reassessment of the intrinsic carrier density temperature dependence in crystalline silicon," Journal of Applied Physics 115, 115-119 (2014).

${ }^{24}$ Sze, S.M., Ng, K.K., Physics of semiconductor devices (John Wiley Sons, INC., 2007).

${ }^{25}$ A. Schenk, "Finite-temperature full random-phase approximation model of band gap narrowing for silicon device simulation," Journal of Applied Physics 84, 3684-3695 (1998).

${ }^{26}$ T. A. Demassa and D. P. Knott, "The prediction of tunnel diode voltage-current characteristics," Solid-State Electronics 13, 131138 (1970).

${ }^{27}$ D. Roy, "On the prediction of tunnel diode IV characteristics," Solid-State Electronics 14, 520-523 (1971).

${ }^{28}$ C. Cohen-Tannoudji, B. Diu, and F. Laloe, Quantum Mechanics, 2 Volume Set (Wiley, 1992).

${ }^{29} \mathrm{ECE}$ Tutorials Electronics and control System, "Tunnel diode working principle and characteristics," .

${ }^{30}$ Y. Yan, "Silicon-based tunnel diode technology," (2008).

${ }^{31}$ H. Schmid, C. Bessire, M. T. Bjrk, A. Schenk, and H. Riel, "Silicon nanowire esaki diodes," Nano Letters 12, 699-703 (2012). 